\title{
Neural correlates of a 'pessimistic' attitude when anticipating events of unknown emotional valence
}

\author{
Uwe Herwig, ${ }^{a, c, *}$ Tina Kaffenberger, ${ }^{a}$ Thomas Baumgartner, ${ }^{\mathrm{b}, \mathrm{d}}$ and Lutz Jäncke ${ }^{\mathrm{b}}$ \\ ${ }^{\text {a } P s y c h i a t r i c ~ U n i v e r s i t y ~ H o s p i t a l ~ Z u ̈ r i c h, ~ S w i t z e r l a n d ~}$ \\ ${ }^{\mathrm{b}}$ Department of Neuropsychology, University of Zürich, Switzerland \\ ${ }^{\mathrm{c}}$ Department of Psychiatry, University of Ulm, Germany \\ ${ }^{\mathrm{d}}$ Institute for Empirical Research in Economics, Laboratory for Neuroeconomics and Social Neuroscience, University of Zürich, Switzerland
}

Received 31 May 2006; revised 27 September 2006; accepted 28 September 2006

\begin{abstract}
Since we do not know what future holds for us, we prepare for expected emotional events in order to deal with a pleasant or threatening environment. From an evolutionary perspective, it makes sense to be particularly prepared for the worst-case scenario. We were interested to evaluate whether this assumption is reflected in the central nervous information processing associated with expecting visual stimuli of unknown emotional valence. While being scanned with functional magnetic resonance imaging, healthy subjects were cued to expect and then perceive visual stimuli with a known emotional valence as pleasant, unpleasant, and neutral, as well as stimuli of unknown valence that could have been either pleasant or unpleasant. While anticipating pictures of unknown valence, the activity of emotion processing brain areas was similar to activity associated with expecting unpleasant pictures, but there were no areas in which the activity was similar to the activity when expecting pleasant pictures. The activity of the revealed regions, including bilateral insula, right inferior frontal gyrus, medial thalamus, and red nucleus, further correlated with the individual ratings of mood: the worse the mood, the higher the activity. These areas are supposedly involved in a network for internal adaptation and preparation processes in order to act according to potential or certain unpleasant events. Their activity appears to reflect a 'pessimistic' bias by anticipating the events of unknown valence to be unpleasant.

(C) 2006 Elsevier Inc. All rights reserved.
\end{abstract}

\section{Introduction}

Being prepared for the future is crucial for survival. The emotional valence of an expected event provides the basis on which our brain develops behavioral strategies that enable us to adapt quickly and efficiently to new and changing circumstances. In

\footnotetext{
* Corresponding author. Department for Psychiatry Research and Psychogeriatric Medicine, Psychiatric University Hospital, GPZ Hegibach, Minervastr. 145, CH-8032 Zürich, Switzerland. Fax: +41 443891414.

E-mail address: uwe.herwig@puk.zh.ch (U. Herwig).

Available online on ScienceDirect (www.sciencedirect.com).
}

everyday life, however, we are often uncertain as to whether a future event will be pleasant or unpleasant. From an evolutionary perspective, it is conceivable that we have come to prepare ourselves for, and thus cope better with, a potentially threatening environment by anticipating the worst case (Darwin, 1872; Fridlund, 1991). We were interested in whether brain activation patterns generated during the expectation of emotional stimuli of unknown valence would reflect this basic assumption. The question is whether expecting events of unknown emotional valence ('unknown' expectation) is associated with activity in brain areas also found during the expectation of events where the valence is known to be positive or negative. Based on the assumption that information processing is biased towards potentially negative events in order to prepare response strategies efficiently for coping with unfavorable consequences, we hypothesized that emotion processing brain areas are activated during 'unknown' expectation which are also activated during expectation of negative events.

From classical psychometric studies, substantial inter-individual differences are known to exist during the expectation of emotional events of unknown valence at least in views ranging from 'optimistic' to 'pessimistic' (Folkman et al., 1986; Picardi et al., 2005a,b). Based on cognitive models of depression suggesting a negative attitude towards future events in depressive subjects (Beck, 1967, 1987), we further hypothesized that, during the anticipation of unknown emotional events, subjects with a higher level of depressiveness would demonstrate stronger activation in brain areas that also process the expectation of negative emotional events.

Based on knowledge about the brain circuitry involved in emotion processing during the expectation of positive or negative emotional stimuli (e.g. Chua et al., 1999; Ploghaus et al., 1999; Sawamoto et al., 2000; Phelps et al., 2001; O'Doherty et al., 2002; Ueda et al., 2003; Wager et al., 2004; Simmons et al., 2004; Bermpohl et al., 2006, Nitschke et al., 2006), distinct brain regions comprising dorsolateral and inferior prefrontal cortex, orbitofrontal cortex, cingulate gyrus, insula, amygdala, thalamus, midbrain, and parieto-occipital areas were principal regions of interest involved in the expectation of emotional stimuli with unknown valence. 
In the context of event-related fMRI measurements, we designed a cueing experiment in which a visual cue signaled forthcoming emotional stimuli (Fig. 1). Four different emotion conditions were used each comprising the expectation (initiated by the cue) and the subsequent presentation of emotional pictures for which the valence was either 'known' (pleasant, unpleasant, neutral) or 'unknown' (random presentation of pictures with either pleasant or unpleasant valence). We compared brain activity during the expectation of the unknown versus the known events. We further correlated the individual activity of the revealed brain regions with ratings of depressiveness, anxiety and personality.

\section{Materials and methods}

Subjects

Sixteen healthy subjects (age 23-36, all right-handed, 10 female) without any history of neurological or psychiatric illness

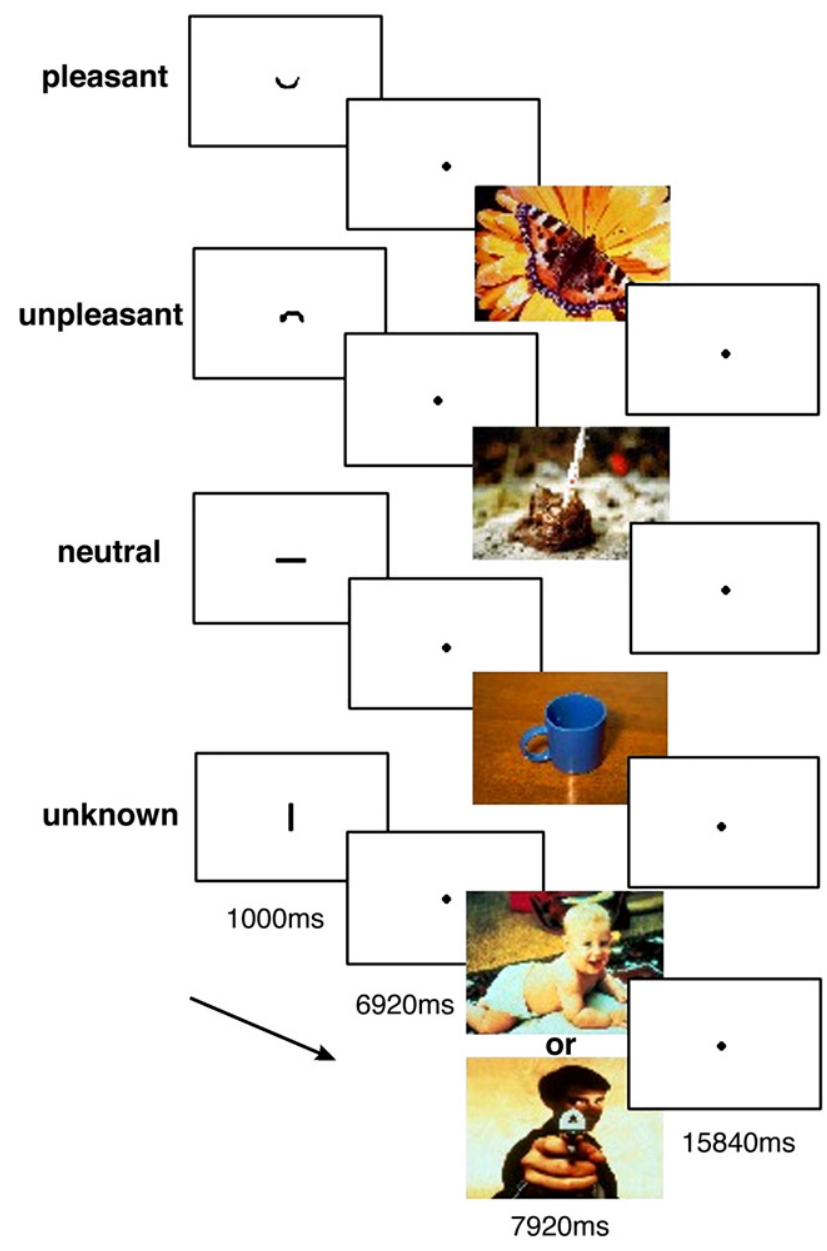

Fig. 1. Experimental task. The four conditions with the respective cues and the durations are presented. The cues, presented for $1000 \mathrm{~ms}$, indicated the valence of the picture which appeared after a delay of further $6920 \mathrm{~ms}$ : " $U$ " prior to a 'pleasant' picture, “ $\cap$ ” prior to an 'unpleasant' picture, "-" prior to a 'neutral' picture, "I" prior to a picture of 'unknown' valence, that could have been pleasant or unpleasant. In the figure, the cues are relatively enlarged for presentation reasons. In the experiment, they were about $1 / 40$ of screen height. and without medication gave written informed consent to take part in this study that was approved by the local ethics committee.

\section{Experimental design}

The subjects performed a task comprising 56 trials with expectation and presentation of emotional pictures while being scanned with fMRI (Fig. 1). Within a trial, the subjects were presented a small cue, depicting either a smiling ('pleasant') " $U$ ", a non-smiling ('negative' or 'unpleasant') " $\cap$ ”, or a neutral symbol "-" announcing the emotional valance of the upcoming picture, or a symbol after which either a pleasant or an unpleasant picture appeared: the 'unknown' condition “|”. The cues were 1/40 of screen height whereas the subsequent pictures filled the screen. On the one hand, the cues were highly abstract and physically comparable, on the other hand, they were intuitively understandable and no prominent working memory component had to be used to establish their meaning. Furthermore, no motor reaction was required, the preparation of which may have interfered with the emotional anticipation.

The cues were presented for $1000 \mathrm{~ms}$ followed by an anticipation period of further $6920 \mathrm{~ms}$, during which a blank screen with a small fixation point was shown. Then pictures of the International Affective Picture System (IAPS, Peter Lang, Miami, USA) (Lang, 1995) of either pleasant, unpleasant or neutral content followed according to the indicating symbol. The pictures were presented for $7920 \mathrm{~ms}$, followed by a baseline of $15,840 \mathrm{~ms}$ to allow the BOLD signal to level off before a new trial started. Altogether, 56 announced pictures were shown, 14 for each condition. Subjects were instructed to expect the stimuli after the cue and to be aware of the indicated emotional valence and then to watch the following picture. The different conditions appeared in a randomized order. The stimuli were matched for complexity and content of faces, scenery, food and nature. Arousal was matched for pleasant and unpleasant stimuli. The task was programmed with Presentation $^{\mathrm{TM}}$ (Neurobehavioral Systems, USA).

\section{fMRI acquisition}

Imaging was performed with a $1.5 \mathrm{~T}$ Siemens Sonata wholebody scanner (Erlangen, Germany) equipped with a head coil. Initially, for each subject, three-dimensional $\mathrm{T} 1 *$ weighted anatomical volumes were acquired (TR/TE 1880/3.22 ms; matrix size $256 \times 256$; slice thickness $1 \mathrm{~mm}$ ) for later coregistration with the fMRI. T2* weighted functional MR images were obtained using echoplanar imaging in an axial orientation. Image size was $64 \times 64$ pixels, with a field of view of $220 \mathrm{~mm}$, flip angle was $90^{\circ}$. One volume covering the whole brain consisted of 22 slices. Slice thickness was $4 \mathrm{~mm}$ with $1 \mathrm{~mm}$ gap resulting in a voxel size of $3.4 \times 3.4 \times 5 \mathrm{~mm}$. Volumes were obtained every $1980 \mathrm{~ms}$ (TE $40 \mathrm{~ms}$ ), altogether 908 volumes, 16 volumes per run. The subjects watched the stimuli in a mirror fixed to the head coil oriented to a screen on which the stimuli were projected by using a video beamer.

\section{fMRI data analysis and statistics}

fMRI data were analyzed using BrainVoyager QX 1.4.9 (Brain Innovation, Maastricht, The Netherlands). The first four images of each functional scan were rejected to allow for T2* equilibration effects. Preprocessing of the functional scans included motion 
correction, slice scan time correction, high frequency temporal filtering, and removal of linear trends. Functional images were superimposed on the 2D anatomical images and incorporated into 3D data sets through trilinear interpolation. The individual 3D data sets were transformed into Talairach and Tournoux space (Talairach and Tournoux, 1988) resulting in a voxel size of $3 \times 3 \times 3 \mathrm{~mm}$ and then spatially smoothed with an $8 \mathrm{~mm}$ Gaussian kernel for subsequent group analysis. Eight predictors, defined to represent the anticipation conditions (negative, pleasant, neutral, unknown) and the presentation conditions (negative, pleasant, neutral, unknown), were used to build the design matrix of the experiment. Expectation period and picture presentation were modeled as an epoch with the canonical hemodynamic response function (HRF) provided by BrainVoyager.

The fMRI data analysis based on the general linear model comprised the following steps: first, fixed effects analyses were calculated separately for each subject for the three contrasts comparing the emotion expectation conditions: (i) negative versus neutral, (ii) pleasant versus neutral, (iii) unknown versus neutral, resulting in summary images. These summary images were subjected to second level random effects analyses (rfx). For the random effects analyses, we set $p<0.005$ and used a cluster threshold of 135 voxel $\grave{a} 1 \times 1 \times 1 \mathrm{~mm}$ as provided by BrainVoyager corresponding to 5 voxel $\grave{a} 3 \times 3 \times 3 \mathrm{~mm}$. This threshold was chosen because most studies in this field of affective neuroscience have used statistical thresholds of similar sizes in order to avoid type-2 errors (e.g. Phelps et al., 2001; Wager et al., 2004). The application of more lenient thresholds in this research field is justified because the hemodynamic responses in the emotional network are weaker than in perception and motor studies due to methodological constraints. The resulting anatomical regions for which the $\mathrm{rfx}$ analysis revealed significantly increased hemodynamic responses during emotional expectation were defined as regions of interest (ROI) for cross-validating the following conjunction analysis.

The next step, addressing our main question and crossvalidating the $\mathrm{rfx}$ results, consisted of fixed effects conjunction analyses on the level $p<0.001$ uncorrected in order to evaluate which of the 'known' emotion expectation conditions negative (ng), positive (ps), and neutral (nt) revealed activations similar to those in the 'unknown' expectation condition. We defined 'unknown' expectation related activity as being similar to a certain 'known' expectation related activity, when both were significantly different from the activations associated with both remaining 'known' emotion expectation conditions. For instance, for the comparison of the 'unknown' and the 'negative' condition, the 'unknown' condition had to differ significantly from 'positive' (contrast exp $\mathrm{uk}>\mathrm{ps}$ ) and 'neutral' (contrast exp uk>nt) related activity, and 'negative' also had to differ from 'positive' (exp ng $>$ ps) and 'neutral' (exp ng $>$ nt) expectation related activity (Fig. 2). These contrasts comprised therefore also the direct comparisons between the 'known' expectation conditions, for instance, 'negative' versus 'positive'.

Accordingly, in order to explore whether the activity of (i) expecting stimuli of unknown emotional events was similar to activity associated with expecting negative stimuli, we analyzed the conjunction: exp $\mathrm{uk}>\mathrm{nt}$ and $\mathrm{uk}>\mathrm{ps}$ and $\mathrm{ng}>\mathrm{nt}$ and $\mathrm{ng}>\mathrm{ps}$, (ii) for the analysis of the relation of 'unknown' and 'positive' expectation: exp $\mathrm{uk}>\mathrm{nt}$ and $\mathrm{uk}>\mathrm{ng}$ and $\mathrm{ps}>\mathrm{nt}$ and $\mathrm{ps}>\mathrm{ng}$, and (iii) for revealing the relation of 'unknown' and 'neutral' expectation: exp $\mathrm{uk}>\mathrm{ng}$ and $\mathrm{uk}>\mathrm{ps}$ and $\mathrm{nt}>\mathrm{ng}$ and $\mathrm{nt}>\mathrm{ps}$.

We performed fixed effects conjunction analyses with separate subject predictors because established random effects conjunction analysis statistics may be subject to error associated with the minimum- $t$ problem (Nichols et al., 2005), according to which areas may be indicated as significant in the conjunction but that are not significant in all single contrasts.

We finally cross-validated the conjunction analyses by verifying that the revealed regions complied with the ROIs derived from the prior more conservative $\mathrm{rfx}$ analyses.

An analysis of the presentation period, which was performed in many previous studies (Phan et al., 2004), and main effect analyses of emotion and expectation were out of the scope of the addressed
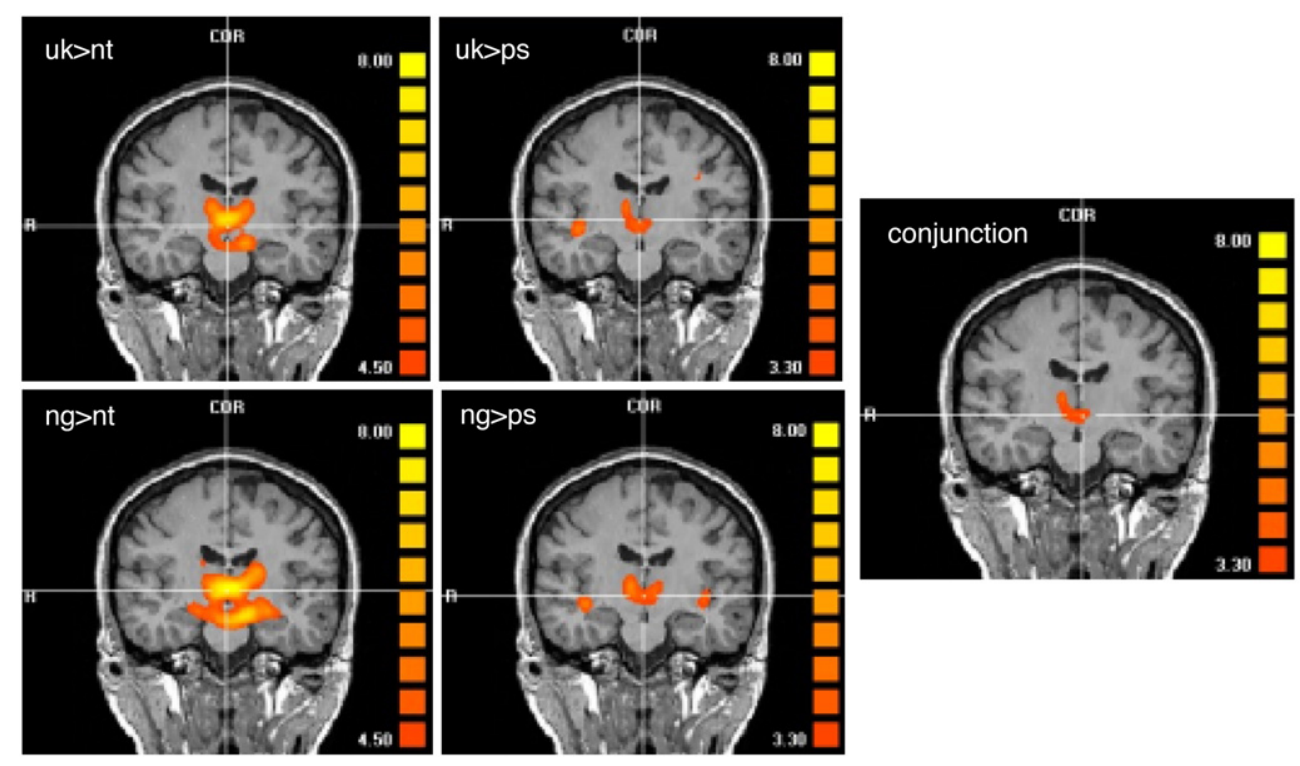

Fig. 2. Example of conjunction analysis. Presented are the activated voxels within the thalamus for the four single contrasts negative $>$ neutral (ng $>$ nt, for presentation reasons here $t>4.5$, in conjunction $t>3.3$ ), negative $>$ positive (ng $>$ ps, $t>3.3$ ), unknown $>$ neutral (uk $>$ nt, here $t>4.5$ ), and unknown $>$ positive (uk $>\mathrm{ps}, t>3.3$ ) that result in the activated voxels within the medial thalamus according to the fixed effects conjunction analysis of these contrasts (conjunction, $t>3.3$; consider as well Fig. 3C). 
hypothesis and were performed on an exploratory basis not reported here. Their results were not in conflict with the reported results. The identification of the anatomical regions was based on the Talairach and Tournoux system (Talairach and Tournoux, 1988), for the thalamus additionally considering a more detailed thalamus specific stereotaxic atlas (Morel et al., 1997) and a report of MRI visualization of the thalamus (Deoni et al., 2005).

\section{Questionnaires and correlation statistics}

The subjects completed a handedness questionnaire (Annett and Bryden), and self-ratings of depressiveness (self-rating depression scale, SDS, German version), anxiety (state trait anxiety inventory, STAI-G, German version), and personality comprising neuroticism and extraversion (Eysenck personality inventory, EPI, Eysenck and Eysenck, 1971). Furthermore, the subjects rated the presented pictures immediately after scanning (presented again as printouts) on a visual analog scale. By using Pearson's correlation, we correlated the individual questionnaire results of the depression scale (depr), the anxiety scale (anx), the neuroticism (neur) and extraversion (ext) scores of the personality inventory with the individual beta weights (mean from all voxels of the respective activated cluster) of the emotion expectation conditions in those regions resulting from fMRI data conjunction analyses. In an attempt to reduce the number of psychometric variables, we calculated a factorial analysis for a possible aggregation of the questionnaires. The inter-correlations (as measured with Pearson correlations) between the questionnaire measures were: depr/neur $r=0.38$, depr/ext $r=-0.01$, depr/anx $r=0.63$, anx/neur $r=0.53$, anx/ext $r=0.23$, neur/ext $r=-0.09$. Although there were two significant correlations, four were non-significant. For instance, neuroticism was reported earlier to correlate with depressiveness (Meites et al., 1980), whenever this was not the case in our sample. Hence, the correlations were too low to warrant the application of data reduction methods like factor analysis, and we relied on the original psychometric variables for the correlation analysis with the beta weights.

\section{Results}

\section{Behavioral analysis}

Sixteen healthy subjects participated in the fMRI experiment. Fourteen were included in the analysis ( 8 female, age 23-36 years), two were excluded because they reported feeling drowsy in the scanner and were unable to concentrate on the task. All subjects were right-handed and had normal, non-pathological ratings concerning depressiveness (self-rating depression scale, SDS, range 27.5-47.5), anxiety (STAI-G, range 22-35), 'neuroticism' (EPI, range 1-10), and 'extraversion' (EPI, range 7-18). After the scanning, the pictures were rated on the 9-step visual analogue scale with 1 being worse, 5 being neutral, and 9 being best. The negative pictures were rated in mean 2.96 , SD 1.35 , the neutral 5.10, SD 0.67, and the positive 7.20, SD 1.25 (mean and standard deviations over all subjects ratings of all pictures).

\section{Brain activation during the expectation of emotional stimuli}

Random effects (rfx) analyses were computed for each subject separately, based on the contrast images obtained for the different emotion expectation conditions. We compared each emotion expectation condition - negative (ng), positive (ps), unknown (uk) - with the neutral (nt) expectation condition.

The analysis of the single contrast expectation negative versus expectation neutral (exp ng $>$ nt, Table 1a) revealed activity within bilateral inferior frontal gyrus (IFG), bilateral insula, anterior cingulate cortex (ACC), bilateral rostral superior temporal gyrus (STG), bilateral 'extended' amygdala, left striatum, bilateral caudate nucleus, bed nucleus of stria terminalis (BedNST), bilateral hippocampal and parahippocampal gyrus, extensively within the thalamus, bilateral midbrain nuclei (covering parts of substantia nigra, nucleus ruber, ventral tegmentum, superior colliculi), left inferior parietal gyrus (IPG), bilateral fusiform gyrus, and bilateral temporo-occipital cortex (TOC).

Analyzing expectation pleasant versus expectation neutral (exp ps $>$ nt, Table 1b) revealed activity within rostral left superior frontal gyrus (SFG), septum, subcallosal cingulate gyrus, bilateral 'extended' amygdala, bilateral hippocampal and parahippocampal gyrus, left striatum, anterior and posterior thalamus, bilateral midbrain nuclei (left substantia nigra, ventral tegmentum), bilateral fusiform gyrus, and right TOC.

Analyzing expectation unknown versus expectation neutral (exp uk>nt, Table 1c) revealed activity within ACC, medial prefrontal cortex, right IFG, bilateral insula, STG, bilateral 'extended' amygdala, left striatum, BedNST, extensively within the thalamus, bilateral midbrain nuclei (focus on nucleus ruber, as well substantia nigra), bilateral fusiform gyrus, and bilateral TOC.

The significantly activated regions revealed by these single random effects contrasts, complying with regions known to be involved in emotion processing according to the literature (Phillips et al., 2003), served as regions of interest (ROI) for the subsequent conjunction analyses in which 'unknown' expectation was compared with the 'known' expectation conditions.

Conjointly activated areas during the 'unknown' and the different 'known' emotion expectation conditions

In order to address our main question, we performed conjunction analyses to uncover which of the 'known' emotion expectation conditions revealed activations similar to those in the 'unknown' expectation condition. Concerning the comparison 'negative' and 'unknown', we revealed activity within bilateral anterior and left posterior insula, right IFG, medial thalamus (covering centromedial, ventromedial and dorsomedial thalamic regions), BedNST, nucleus ruber, and right TOC (Table 2, Fig. 3). These areas fitted with the ROIs derived from the $\mathrm{rfx}$ analysis. The equivalent analysis of the relation of 'unknown' and 'positive' expectation did not deliver any results even at a level of $p<0.01$ uncorrected. The analysis for revealing the relation of 'unknown' and 'neutral' expectation showed activity in the right MFG BA 9/46 ( $t$-max 5.4), but no further activity even at a level of $p<0.01$ uncorrected. Hence, we found that distinct brain regions activated by 'unknown' expectation were also activated by 'negative' expectation, but not by 'positive' expectation. In contrast to these regions, other regions as for instance the amygdala were active in all emotion expectation conditions versus neutral expectation (Fig. 4) as reflected by the random effects single contrasts.

\section{Correlation with psychometric questionnaires}

The suggestion was made that subjects with a higher level of depressiveness may exert stronger activity in areas associated with 
Table 1

fMRI analysis of main contrasts and emotion expectation contrasts

\begin{tabular}{|c|c|c|c|c|c|c|}
\hline \multirow[t]{2}{*}{ Anatomic regions } & \multirow{2}{*}{$\begin{array}{l}\text { Brodmann } \\
\text { area }\end{array}$} & \multirow{2}{*}{$\begin{array}{l}\text { Voxel } \\
\mathrm{mm}^{3}\end{array}$} & \multicolumn{3}{|c|}{ Talairach coordinates } & \multirow[t]{2}{*}{$t$-max } \\
\hline & & & $x$ & $y$ & $z$ & \\
\hline \multicolumn{7}{|l|}{ (a) Expectation negative $>$ neutral } \\
\hline Inferior frontal gyrus $\mathrm{L}>\mathrm{R}$ & 45 & 1566 & -31 & 19 & 11 & 5.8 \\
\hline Anterior insula blt & 13 & 798 & -33 & 8 & 2 & 7.9 \\
\hline Posterior insula L & 13 & 972 & -31 & 1 & -5 & 4.6 \\
\hline Anterior/medial cingulate gyrus & $24 / 32$ & 456 & 1 & 17 & 19 & 4.2 \\
\hline Superior temporal gyrus $\mathrm{L}>\mathrm{R}$ & 38 & 916 & -31 & 7 & -16 & 6.4 \\
\hline Extended amygdala blt & 19 & 860 & -23 & -3 & -9 & R 6.8, L 6.4 \\
\hline Thalamus $\mathrm{L}>\mathrm{R}$ & & 5022 & -12 & -8 & 11 & $6.7^{`}$ \\
\hline Bed nucleus of stria terminalis $\mathrm{R}>\mathrm{L}$ & & 583 & 10 & 2 & 8 & 5.3 \\
\hline Nucleus caudatus $\mathrm{R}>\mathrm{L}$ & & 766 & 13 & 1 & 20 & 7.3 \\
\hline Striatum L & & 1133 & -13 & 0 & 11 & 5.3 \\
\hline Parahippocampal gyrus $\mathrm{R}>\mathrm{L}$ & $28 / 35$ & 322 & 23 & -18 & -8 & R.5.8 L.4.3 \\
\hline Midbrain nuclei $\mathrm{L}>\mathrm{R}$ & & 1115 & -7 & -16 & -12 & L $7.3, \mathrm{R} 6.1$ \\
\hline Inferior parietal gyrus & 40 & 956 & -58 & -29 & 28 & 6.5 \\
\hline Fusiform gyrus $\mathrm{L}>\mathrm{R}$ & $19 / 37$ & 1460 & -32 & -66 & -11 & 9.6 \\
\hline Temporo-occipital cortex $\mathrm{R}>\mathrm{L}$ & $19 / 37$ & 2696 & 47 & -63 & 0 & 7.6 \\
\hline \multicolumn{7}{|l|}{ (b) Expectation positive $>$ neutral } \\
\hline Superior frontal gyrus L & $9 / 10$ & 2917 & -5 & 54 & 24 & 5.8 \\
\hline Septum & 2721 & 2721 & 1 & 19 & 15 & 6.7 \\
\hline Subcallosal cingulate gyrus & 25 & 686 & -2 & 8 & -1 & 7.3 \\
\hline Extended amygdala $\mathrm{R}>\mathrm{L}$ & & 663 & 19 & -3 & -9 & R 6.8, L 5.3 \\
\hline Parahippocampal gyrus $\mathrm{L}>\mathrm{R}$ & $28 / 35$ & 2002 & -19 & -18 & -9 & L 9.6, R 7.0 \\
\hline Posterior thalamus/pulvinar & & 1413 & -2 & -28 & 5 & 6.0 \\
\hline Midbrain nuclei $\mathrm{L}>\mathrm{R}$ & & 426 & -11 & -25 & -12 & 5.1 \\
\hline Fusiform gyrus $\mathrm{R}>\mathrm{L}$ & 19 & 647 & 35 & -67 & -11 & 7.2 \\
\hline Temporo-occipital cortex $\mathrm{R}$ & $19 / 37$ & 1457 & 47 & -64 & -1 & 7.3 \\
\hline \multicolumn{7}{|l|}{ (c) Expectation unknown > neutral } \\
\hline Anterior cingulate gyrus $\mathrm{R}>\mathrm{L}$ & $24 / 32$ & 2206 & 4 & 20 & 29 & 7.0 \\
\hline Medial frontal cortex $\mathrm{R}$ & $8 / 9$ & 299 & 6 & 38 & 34 & 5.7 \\
\hline Inferior frontal gyrus $\mathrm{R}$ & 45 & 237 & 45 & 24 & 12 & 4.9 \\
\hline Anterior insula blt $\mathrm{L}>\mathrm{R}$ & 13 & 1604 & -30 & 18 & 1 & 5.3 \\
\hline Posterior insula L & 13 & 496 & -35 & 0 & -8 & 5.4 \\
\hline Superior temporal gyrus blt & 38 & 1479 & -35 & 10 & -16 & 5.5 \\
\hline Extended amygdala $\mathrm{R}>\mathrm{L}$ & & 400 & 19 & -3 & -8 & 7.0 Fig. 4 \\
\hline Striatum L & & 672 & -13 & -3 & 5 & 4.2 \\
\hline Bed nucleus of stria terminalis & & 493 & 9 & 2 & 9 & 5.6 \\
\hline Thalamus $\mathrm{L}>\mathrm{R}$ & & 2611 & -2 & -20 & 2 & 6.5 \\
\hline Midbrain nuclei $\mathrm{R}>\mathrm{L}$ & & 984 & 5 & -24 & -8 & 5.5 \\
\hline Temporo-occipital cortex $\mathrm{R}>\mathrm{L}$ & $19 / 37$ & 3653 & 46 & -61 & 0 & 8.7 \\
\hline Posterior superior temporal gyrus $\mathrm{R}$ & $22 / 38$ & 1362 & 34 & 6 & -12 & 4.7 \\
\hline Fusiform gyrus L $>\mathrm{R}$ & $19 / 37$ & 454 & -33 & -60 & -15 & 4.9 \\
\hline
\end{tabular}

Activated regions according to the random effects analyses of the contrasts: (a) expectation negative versus neutral, (b) expectation positive versus neutral, (c) expectation unknown versus neutral. Indicated are the amounts of voxels in $\mathrm{mm}^{3}$, the Talairach coordinates $(x, y$, $z$ ) of the center of mass of the activation, and the maximal $t$-value of the voxels within each region.

'unknown' and 'negative' expectation. We tested this hypothesis by correlating (Pearson) the questionnaire data obtained from the self-rating depression scale (SDS), state/trait anxiety inventory (STAI-G), the 'neuroticism' scale (reflecting emotional instability concerning depressiveness, anxiety, and low self-esteem), and the 'extraversion' scale of the Eysenck personality inventory (EPI, Eysenck and Eysenck, 1971; Gray, 1972), with the mean beta weights obtained from the significantly activated clusters of the aforementioned conjunction analyses. This analysis revealed significant correlations between the activity in right IFG, medial thalamus, and right anterior and left posterior insula, thus the major part of the identified regions, during 'unknown' expectation and both the 'depressiveness' and 'neuroticism' scores (Fig. 3 and Table 3). The activity in medial thalamus and nucleus ruber further correlated with the anxiety score.

\section{Discussion}

The main purpose of our study was to delineate characteristics of brain activity associated with expecting events of 'unknown' emotional valence compared with expecting events of 'known' valence. Complying with predefined ROIs, we found activity in medial thalamus, right anterior and left posterior insula, right IFG, 
Table 2

Conjunction analyses of the emotion expectation contrasts

\begin{tabular}{|c|c|c|c|c|c|c|c|}
\hline \multirow[t]{2}{*}{ Anatomic regions } & \multirow{2}{*}{$\begin{array}{l}\text { Brodmann } \\
\text { area }\end{array}$} & \multirow{2}{*}{$\begin{array}{l}\text { Voxel } \\
\text { size }\end{array}$} & \multicolumn{3}{|c|}{ Talairach coordinates } & \multirow[t]{2}{*}{$t$-max } & \multirow[t]{2}{*}{ Figure } \\
\hline & & & $x$ & $y$ & $z$ & & \\
\hline \multicolumn{8}{|c|}{ Expectation unknown and negative $>$ positive and neutral } \\
\hline Inferior frontal gyrus $\mathrm{R}$ & 45 & 610 & 47 & 17 & 16 & 4.2 & Fig. 3A \\
\hline Inferior frontal gyrus/anterior insula $\mathrm{R}$ & 13 & 73 & 37 & 21 & -4 & 3.9 & \\
\hline Anterior insula L & 13 & 763 & -29 & 19 & 8 & 4.4 & \\
\hline Posterior insula L & 13 & 131 & -36 & -2 & -4 & 4.3 & 3B \\
\hline Bed nucleus of stria terminals $\mathrm{R}$ & & 205 & 11 & 1 & 8 & 3.9 & \\
\hline Medial thalamus bilateral & & 1177 & 5 & -14 & 4 & 4.6 & $3 \mathrm{C}$ \\
\hline Nucleus ruber $\mathrm{R}$ & & 121 & 7 & -20 & -8 & 4.0 & $3 \mathrm{D}$ \\
\hline Temporo-occipital cortex L & 19 & 1188 & -48 & -62 & -11 & 4.8 & \\
\hline
\end{tabular}

Activated regions according to the conjunction analysis of expectation 'unknown' and 'negative' stimuli, both versus expecting 'positive' and 'neutral' stimuli. Indicated are the amounts of voxels in $\mathrm{mm}^{3}$, the Talairach coordinates $(x, y, z)$ of the center of mass of the activation, and the maximal $t$-value of the voxels within the regions.

and nucleus ruber during the 'unknown' expectation being similar to the 'unpleasant' expectation that additionally correlated with the individual degree of 'depressiveness' and 'neuroticism' (Eysenck and Eysenck, 1971). Similar activity during the 'unknown' and the 'negative' condition was further found within the left anterior insula, bed nucleus of the stria terminalis, and left occipitotemporal cortex. No areas were found with similar activity during the 'unknown' and the 'positive' condition, and only one area, within the right MFG, showed comparable activity for the 'unknown' and the 'neutral' condition. Thus, the activation pattern in distinct brain regions during the 'unknown' expectation condition was similar to the activation pattern found during the explicit expectation of negative events. This finding supports our hypothesis that events of unknown emotional valence to a certain extent are processed as if they are expected negative events and not expected positive events.

\section{Anatomical and functional features of the revealed areas}

The medial thalamic region that was associated with negative and unknown expectation comprised areas within the ventromedial, dorsomedial, and centromedial thalamus (Talairach and Tournoux, 1988; Morel et al., 1997; Deoni et al., 2005). Medial thalamic regions receive input from viscerosensitive and pain mediating brainstem areas such as the parabrachial nucleus, the subnucleus reticularis, and the periaqueductal gray (Vogt, 2005; Craig, 2002). The ventromedial thalamus is considered to be a relay within the visceroceptive pathway to posterior and anterior insula (Craig, 2002). The centromedial thalamus projects to the ACC, insula, and amygdala (Augustine, 1996; Collins and Pare, 1999; Vogt, 2005; Minamimoto et al., 2005), the dorsomedial thalamus, as well to the insula and the ACC, both supposedly for emotional-motivational functions (Heimer, 2003; Allen et al., 1991).

Insular regions were also prominently active during 'unknown' and 'negative' expectation. This is of particular interest given the increasing importance of the insula in concepts of emotion processing. Insular regions have a wide range of reciprocal connections to prefrontal areas, ACC, thalamus, amygdala, hypothalamus, and to brainstem regions as the parabrachial nucleus for relaying visceral afferents (Augustine, 1996; Yasui et al., 1991). Furthermore, the insula receives input from the aforementioned medial thalamic regions (Augustine, 1996; Allen et al., 1991). On the functional level, the insula was found to be involved in the anticipation of electric shocks (Chua et al., 1999), noxious thermal stimuli (Ploghaus et al., 1999), fear indicating stimuli together with the amygdala (Phelps et al., 2001), and of aversive pictures (Simmons et al., 2004). Furthermore, the insula was found to be involved in differential positive versus negative emotion processing (Buchel et al., 1998), particularly in fearful face processing (Morris et al., 1998), in pain perception (Peyron et al., 2000), in judgments about emotions (Gorno-Tempini et al., 2001), and, particularly the right anterior insula, in emotional states such as anger, disgust, sexual arousal, and subjective feeling of trustworthiness (Phillips et al., 1997; Stoleru et al., 1999; Winston et al., 2002). Accordingly, initial reports of the insula as being associated with disgust (Phillips et al., 1997) were modified towards concepts attributing a more general role in emotion processing (Paulus et al., 2005). It was proposed that the physical sensation of emotional responses is dependent on sensations from the viscera represented in the insula (Damasio, 1996, 2000; Dunckley et al., 2005). The insula may form an interoceptive cortical image for the subjective evaluation of the body's physiological condition (Bechara et al., 1997; Critchley et al., 2001). In this context, the insula may be involved in the mediation of somatic markers (Damasio, 1996) in the sense of bodily viscerosensitive signals for decision making and behavioral planning according to emotional valences. This representation may be associated with the ability to perceive the self as a physical and separate entity, corresponding to 'self-awareness', thereby integrating information about past experiences and future goals (Craig, 2002; Churchland, 2002).

Furthermore, we found the right IFG within the ventrolateral prefrontal cortex (VLPFC) to be associated with 'negative' and 'unknown' expectation. The IFG was previously reported as being involved in emotion processing during the expectation of negative stimuli (Ueda et al., 2003), the perception of fearful stimuli (Pessoa, 2005), the inhibition of positive attitudes (Wood et al., 2005), and the detection of behaviorally relevant stimuli (Corbetta and Shulman, 2002). It was further reported to be active during visual attention (Pessoa et al., 2003), and it may be involved in a top-down activation of the temporo-occipital associative visual areas that we also found to be activated with 'negative' and 'unknown' expectation. The IFG was supposed to represent multi-modal sensory cues with high emotional salience (Yamasaki et al., 2002) and to be involved in emotional-cognitive integration (Mayberg, 

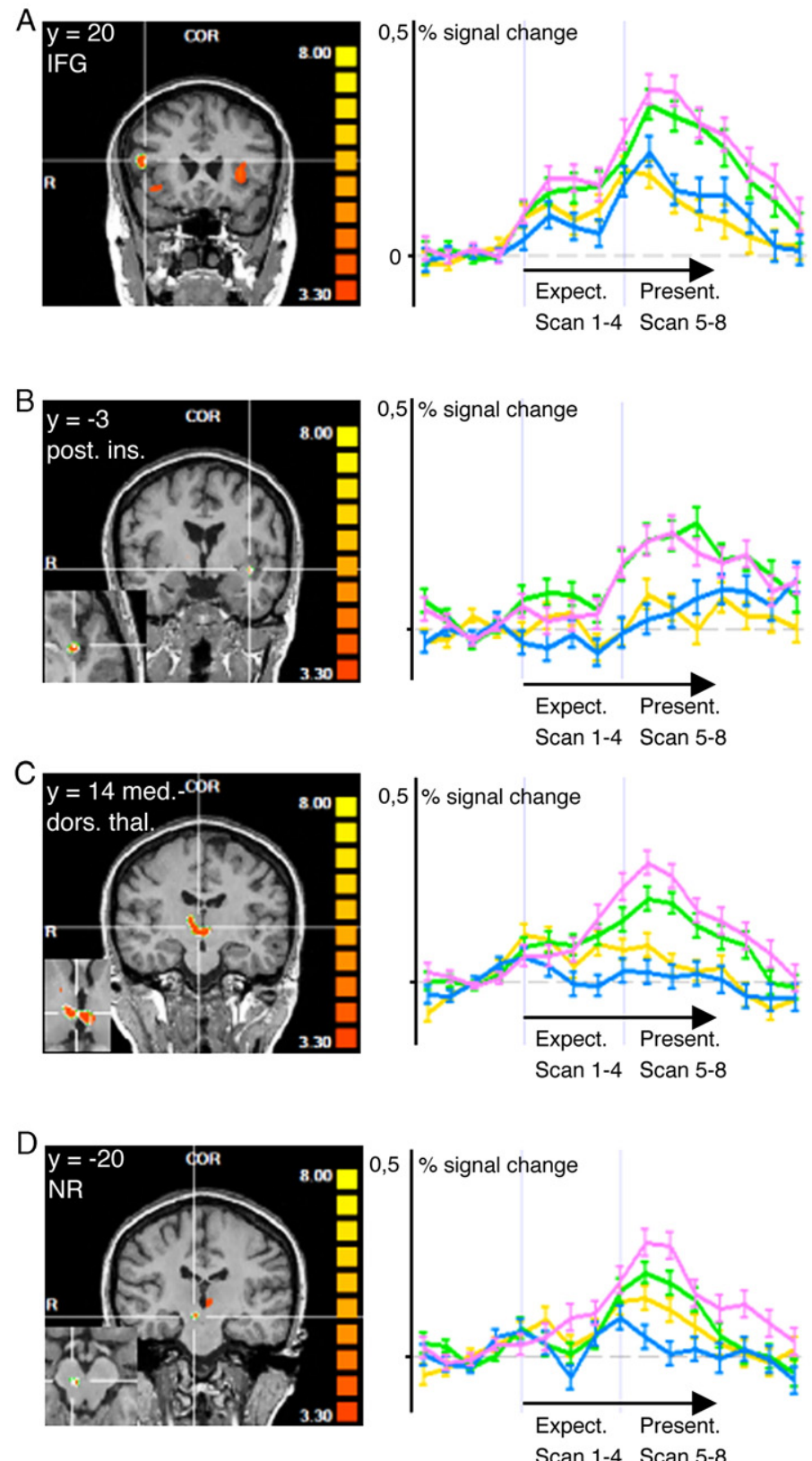
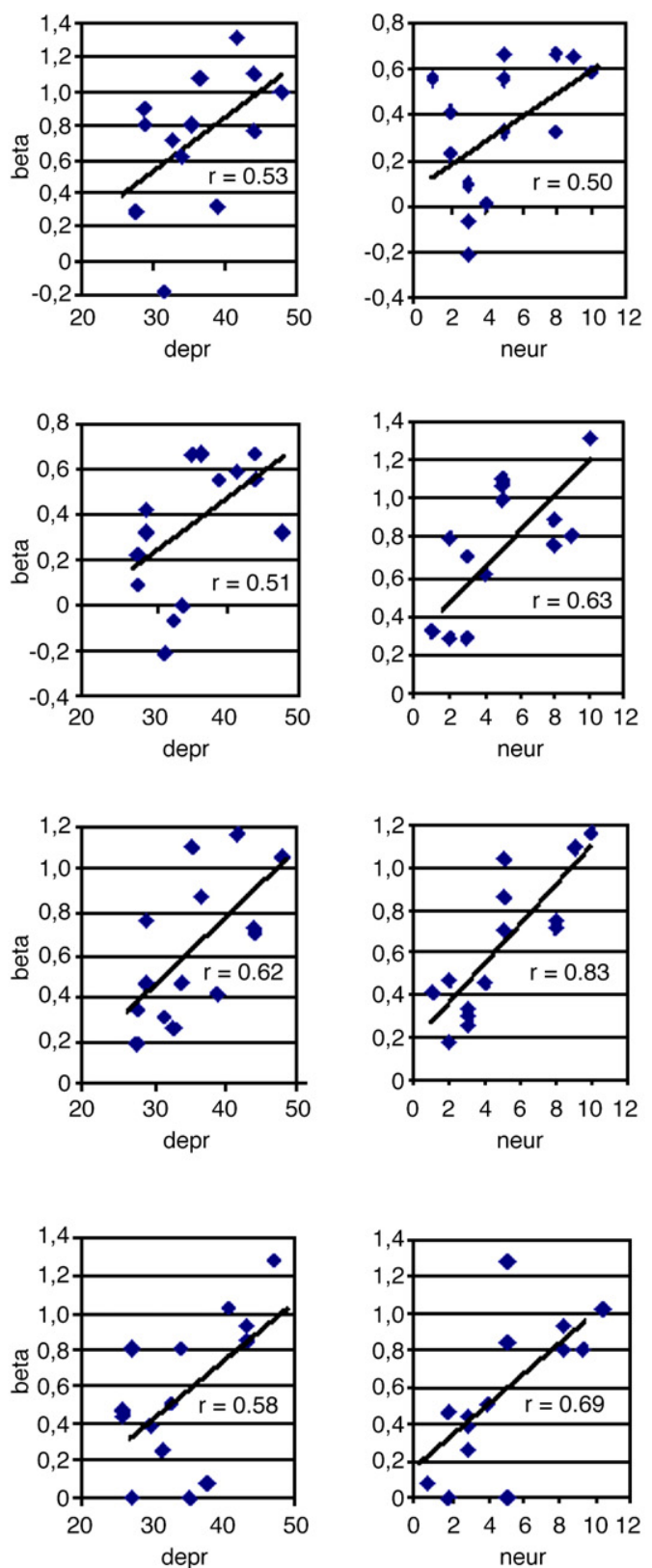

Fig. 3. Comparison of 'known' and 'unknown' expectation. Conjunction analysis of the conditions 'unknown' and 'unpleasant' expectation versus 'pleasant' and 'neutral'. The activated voxels were coregistered with a structural MRI and color coded according to their significance ( $t$-value). (A) Right inferior frontal gyrus, (B) left posterior insula, (C) medial thalamus, and (D) right nucleus ruber (NR), shown in the coronal slice, and a cut-out of the axial slice of insula, thalamus, and NR in the lower left of the respective pictures. Furthermore, on the right, the time courses of the activities within the marked ROIs are shown in percent BOLD signal change, indicating the conjoint activity of the unpleasant expectation with the unknown expectation. The vertical gray bars represent the beginning of the expectation and presentation periods comprising each 4 volumes. When interpreting the time courses, the delayed hemodynamic response function has to be considered. Furthermore, the correlations of the beta weights of the activity during the 'unknown' expectation condition with the 'depressiveness' (depr, selfrating depression scale) and the 'neuroticism' (neur, Eysenck personality inventory) scores are presented ( $r=$ Pearson's correlation coefficient, $r=0.50$ and higher corresponds to $p<0.05$ ). Abbreviations: IFG, inferior frontal gyrus, R, right, Expect., expectation, Present., presentation, depr, depressiveness score, neur neuroticism score, $r$, Pearson correlation coefficient, post., posterior, ins., insula, dors., dorsal, thal., thalamus, NR, nucleus ruber.

2003). In some respect, the right non-dominant IFG may be seen as an emotional counterpart to the left-sided IFG, which is known to be involved in verbal processing (Zurowski et al., 2002).

At the brainstem level, we found the nucleus ruber (NR) to be associated with 'negative' and 'unknown' expectation. The NR was suggested as being involved in psychomotor modulation and in behavioral attendance (Horn et al., 2002). During expectation, it may prime or facilitate the readiness for a later motor reaction. Finally, a region covering the bed nucleus of the stria terminalis was activated. This region was considered to form a functional unit with the 'extended' amygdala (Heimer, 2003) and may be a link to further emotion processing circuits. 

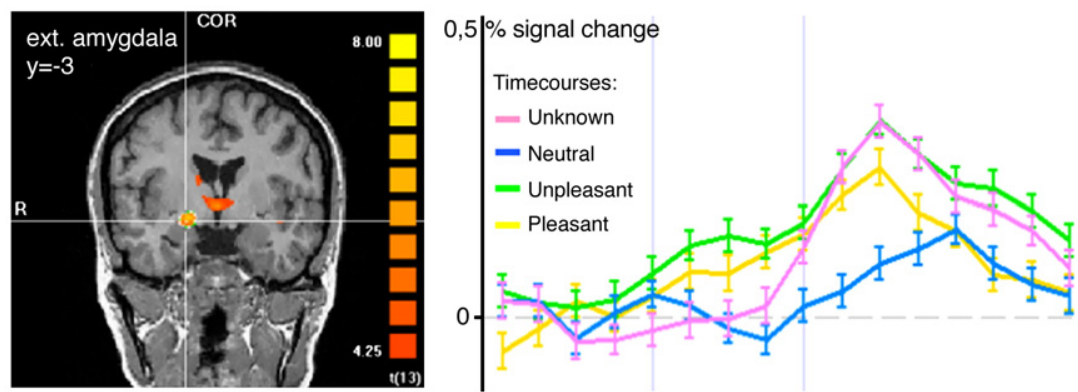

Fig. 4. Expectation related activity in all emotion conditions: amygdala. Activated voxels of right sublenticular extended (ext.) amygdala, random effects analysis contrast 'unknown expectation' versus 'neutral expectation', threshold presented here $p<0.0001$, results in Table 2 refer to $p<0.001$. This region was active in all emotion conditions, consider Tables $2 \mathrm{a}-\mathrm{c}$, as is indicated also by the time courses.

According to the ROIs derived from the single random effects analysis contrasts which are in line with recent findings (Nitschke et al., 2006; Bermpohl et al., 2006; Herwig et al., in press), we suggested the ACC to be active in the conjunction analyses for unpleasant and unknown expectation, but it was not. However, in the rfx single contrasts, an area within ACC covering parts of BA 24/32 was active during expectation of the unpleasant and the unknown compared to neutral events. The ACC receives prominent input from medial thalamic regions and is supposed to be involved in conflict monitoring between functional state and perceived new information with potential affective or motivational consequences (Carter et al., 2000; Vogt, 2005). Particularly, its affective ventral subdivision (Bush et al., 2000) is suggested to be the point of integration of visceral, attentional, and emotional information for regulation of affect and to be involved in top-down control (Dalgleish, 2004). It may thus be involved in a higher level representation of bodily states (Damasio et al., 2000; Critchley et al., 2001) and general emotionalcognitive integration (Mayberg, 2003). Therefore, we suggest that the ACC interacts closely with the other mentioned areas during expectation-related emotion processing.

Another important region within emotion processing, the amygdala, was activated in all three random effects single contrasts of the emotion expectation conditions versus neutral. This underlines views of its function of a more general role in emotion processing as for emotional arousal or intensity without a valence specificity (Baxter and Murray, 2002; Anderson et al., 2003; Gottfried et al., 2003; McClure et al., 2004).
Taken together, we found evidence for a 'medial-thalamicinsular-inferior-frontal-rubral' circuit associated with expecting events of unknown emotional valence, the activity of which resembled the expectation of negative events and also correlated with individual depressiveness. The revealed areas are consistent with the proposed 'ventral system' of emotion processing (Phillips et al., 2003) for identification of the emotional significance of a stimulus, production of an affective state, and autonomic response regulation.

\section{'Pessimistic' expectation?}

The fMRI results of similar activation during unknown and negative expectation were supported by the finding that the activity of medial thalamus, right anterior and left posterior insula, right IFG, and nucleus ruber during expectation of the 'unknown' emotion correlated positively with the 'depressiveness' and with the 'neuroticism' scores: the higher the 'depressiveness' and the 'neuroticism' scores, the higher the activity in the abovementioned brain areas. Concerning a descriptive comparison, there were on the other hand no correlations of these areas with 'depressiveness' or 'neuroticism' during 'negative' expectation. This lends further support to our hypothesis of 'pessimistic' activity during 'unknown' expectation because during expectation of certainly negative events the subjects knew that a negative event would occur. Therefore, they had no choice between preparing for a

Table 3

Correlation of beta weights with rating scores

\begin{tabular}{|c|c|c|c|c|c|c|c|c|c|c|c|c|c|c|c|c|}
\hline & \multicolumn{2}{|l|}{ uk } & \multicolumn{2}{|l|}{ ng } & \multicolumn{2}{|l|}{ ps } & \multicolumn{2}{|l|}{$\mathrm{nt}$} & \multicolumn{2}{|l|}{ uk } & \multicolumn{2}{|l|}{ ng } & \multicolumn{2}{|l|}{ ps } & \multicolumn{2}{|l|}{$\mathrm{nt}$} \\
\hline & depr & anx & depr & anx & depr & anx & depr & anx & neur & extr & neur & extr & neur & extr & neur & extr \\
\hline \multicolumn{17}{|c|}{ Conjunction of expectation unknown and negative $>$ positive and neutral } \\
\hline IFG R & 0.53 & 0.26 & 0.25 & 0.25 & 0.29 & 0.13 & 0.64 & 0.37 & 0.63 & 0.41 & 0.19 & 0.28 & 0.21 & 0.04 & 0.45 & -0.34 \\
\hline IFG/anterior insula $\mathrm{R}$ & 0.55 & 0.26 & 0.26 & 0.32 & 0.23 & 0.32 & 0.43 & 0.32 & 0.67 & 0.29 & 0.33 & 0.15 & 0.15 & -0.11 & 0.31 & -0.49 \\
\hline Ant. insula L & 0.42 & 0.12 & 0.43 & 0.32 & 0.34 & 0.38 & 0.41 & 0.34 & 0.44 & -0.03 & 0.26 & 0.22 & 0.35 & -0.30 & 0.34 & -0.40 \\
\hline Post. insula L & 0.51 & 0.25 & 0.43 & 0.30 & 0.17 & 0.23 & 0.17 & 0.01 & 0.50 & -0.15 & 0.15 & 0.15 & 0.21 & -0.12 & 0.06 & -0.22 \\
\hline Bed nucleus stria terminalis $\mathrm{R}$ & 0.29 & 0.28 & -0.18 & -0.11 & 0.30 & 0.25 & 0.06 & 0.04 & 0.40 & 0.26 & -0.02 & 0.30 & 0.24 & -0.22 & -0.16 & 0.08 \\
\hline Med. thalamus blt & 0.62 & 0.59 & 0.23 & 0.44 & 0.22 & 0.30 & 0.10 & 0.25 & 0.83 & 0.11 & 0.12 & 0.35 & 0.16 & -0.23 & 0.22 & -0.45 \\
\hline Red nucleus R & 0.58 & 0.59 & 0.41 & 0.48 & 0.39 & 0.51 & 0.24 & 0.26 & 0.69 & 0.18 & 0.32 & 0.28 & 0.32 & -0.20 & 0.35 & -0.26 \\
\hline Temp-occ L & 0.51 & 0.08 & 0.53 & 0.22 & 0.28 & 0.02 & -0.27 & -0.35 & 0.22 & 0.22 & 0.17 & 0.15 & 0.28 & -0.10 & 0.06 & 0.00 \\
\hline
\end{tabular}

Correlation of beta weights of the regions associated with the conjunction 'unknown and negative versus neutral and positive' with the rating scores. Pearson's $r=0.5$ and higher correspond to $p<0.05$ two-tailed, significant correlations indicated in bold. Abbreviations: uk, unknown, ng, negative, ps, positive, nt, neutral expectation conditions, depr, depression score, anx, anxiety score, neur, neuroticism score, extr, extraversion score, IFG, inferior frontal gyrus, R, right, L, left, ant., anterior, post., posterior, med., medial, blt, bilateral, NR, nucleus ruber, temp-occ, temporo-occipital. 
pleasant or unpleasant event, and a 'pessimistic' attitude would have had no biasing influence on brain activity. Furthermore, when facing unknown events, the aspect of unpredictability may lead to unpleasantness contributing to the revealed similar activity, whereby the emotional response to unpredictable situations depends on several modulator variables as e.g. personality traits (Ainslie, 2003; Barrett and Armony, 2006). On the other hand, the appraisal of uncertainty as an element of fear when expecting negative events (Smith and Ellsworth, 1985) may account for the similarity of unknown and negative expectation as well. Apart and generally, the correlations with the ratings support the suggestion that the revealed fMRI activity was emotion processing specific and that this activity was not likely to be for instance solely arousal-related.

Our results are consistent with the view of brain activity reflecting a 'pessimistic' or 'cautious' bias toward future events. In this context, we could not determine any brain activity that could be regarded as reflecting an 'optimistic' attitude by exerting similar activity during the 'unknown' and the 'positive' condition. People with higher activity within the revealed circuit and with more depressed mood who do not know what the future holds may estimate an 'unknown' and uncertain future as even more unpleasant than others and, to their advantage, may be better prepared when negative events indeed do occur.

Generally, the influence of internal psychological attitudes on brain activity when facing new events has also been described in other studies: the expectation of pain enhanced responses to nonpainful sensory stimuli in the ACC, posterior insula, and parietal operculum (Sawamoto et al., 2000). Placebo induced an increase of activity in midbrain and prefrontal areas during anticipation of thermal pain (Wager et al., 2004). Subjective expectations of less pain than objectively applied reduced activity in somatosensory cortex, insula, and ACC (Koyama et al., 2005).

The revealed correlations with depressiveness are of importance concerning possible pathophysiological aspects of psychiatric disorders such as depression. The cognitive function of expectation has been described as dysfunctional in depression and was interpreted within the cognitive triade as a negative cognitive bias towards the future (Beck, 1967, 1987). Furthermore, the state of depressiveness is often concomitant with unpleasant somatic feelings and a negative self perception, which may be associated with a dysregulation of interoceptive areas such as the insula (Davidson et al., 2003). These may induce subjective unpleasantness due to misinterpretation of cues based on unpleasant previous experiences. Our results emphasize an important role of unpleasant viscero-somatic feelings and their central nervous processing in contributing to depressiveness, in the sense of dysfunctional somatic signals. Depressiveness-related activity in medial thalamic, insular, and right IFG regions may be further investigated as potential neurobiological markers for depressed states.

The finding of a circuit for biasing the organism towards potentially threatening events makes evolutionary sense. The chances of survival of our early ancestors who for instance responded to rustling sounds in the undergrowth as a sign of impending attack by a predator were conceivably higher than of those who did not. In this context, one may guess that a certain degree of depressiveness may be of evolutionary advantage. Interestingly, some of the involved brain regions may represent specific emotion expectation processing in humans as parts of the ventromedial thalamus were found to be more prominent in humans compared to lower primates, and certain cytological features in anterior insula and ACC were found only in humans but not in primates (Nimchinsky et al., 1999; Craig, 2002).

In conclusion, the similarity of 'unknown' and 'unpleasant' expectation-related brain activity may be attributed to the evolved advantage for the individual's chances of survival of preparing itself for the occurrence of expected events of unknown valence in a similar way as for known, potentially threatening events. As the activity of the revealed brain regions, particularly medial thalamus, insula, right IFG, and nucleus ruber, correlated with the individual subjects' depressiveness, they are suggested as being involved in biasing the organism towards preparation for the worst case. Principally, brain activity during the expectation of events of unknown valence appeared to reflect an underlying 'pessimistic' or 'cautious' bias as if the expected events will be unpleasant.

\section{Acknowledgments}

We are grateful to Dr. D. Huber and C. Sauerwald, Hirslanden Clinic, Zürich for providing the fMRI scanning.

$\mathrm{UH}$ and TK contributed equally to this work.

\section{References}

Allen, G.V., Saper, C.B., Hurley, K.M., Cechetto, D.F., 1991. Organization of visceral and limbic connections in the insular cortex of the rat. J. Comp. Neurol. 311, 1-16.

Ainslie, G., 2003. Uncertainty as wealth. Behav. Processes 64 (3), 369-385. Anderson, A.K., Christoff, K., Stappen, I., Panitz, D., Ghahremani, D.G., Glover, G., Gabrieli, J.D., Sobel, N., 2003. Dissociated neural representations of intensity and valence in human olfaction. Nat. Neurosci. 6, 196-202.

Augustine, J.R., 1996. Circuitry and functional aspects of the insular lobe in primates including humans. Brain Res. Brain Res. Rev. 22, 229-244.

Barrett, J., Armony, J.L., 2006. The influence of trait anxiety on autonomic response and cognitive performance during an anticipatory anxiety task. Depress. Anxiety 23 (4), 210-219.

Baxter, M.G., Murray, E.A., 2002. The amygdala and reward. Nat. Rev., Neurosci. 3, 563-573.

Bechara, A., Damasio, H., Tranel, D., Damasio, A.R., 1997. Deciding advantageously before knowing the advantageous strategy. Science 275, 1293-1295.

Beck, A.T., 1967. Depression: Clinical, Experimental and Theoretical Aspects.

Beck, A.T., 1987. Cognitive models of depression. J. Cogn. Psychother.: Int. Q. 5-37.

Bermpohl, F., Pascual-Leone, A., Amedi, A., Merabet, L.F., Fregni, F., Gaab, N., Also, D., Schlaug, G., Northoff, G., 2006. Dissociable networks for the expectancy and perception of emotional stimuli in the human brain. NeuroImage 30, 588-600.

Buchel, C., Morris, J., Dolan, R.J., Friston, K.J., 1998. Brain systems mediating aversive conditioning: an event-related fMRI study. Neuron 20, 947-957.

Bush, G., Luu, P., Posner, M.I., 2000. Cognitive and emotional influences in anterior cingulate cortex. Trends Cogn. Sci. 4, 215-222.

Carter, C.S., Macdonald, A.M., Botvinick, M., Ross, L.L., Stenger, V.A., Noll, D., Cohen, J.D., 2000. Parsing executive processes: strategic vs. evaluative functions of the anterior cingulate cortex. Proc. Natl. Acad. Sci. U. S. A. 97, 1944-1948.

Chua, P., Krams, M., Toni, I., Passingham, R., Dolan, R., 1999. A functional anatomy of anticipatory anxiety. NeuroImage 9, 563-571.

Churchland, P.S., 2002. Self-representation in nervous systems. Science 296, 308-310.

Collins, D.R., Pare, D., 1999. Reciprocal changes in the firing probability of lateral and central medial amygdala neurons. J. Neurosci. 19, 836-844. 
Corbetta, M., Shulman, G.L., 2002. Control of goal-directed and stimulusdriven attention in the brain. Nat. Rev., Neurosci. 3, 201-215.

Craig, A.D., 2002. How do you feel? Interoception: the sense of the physiological condition of the body. Nat. Rev., Neurosci. 3, 655-666.

Critchley, H.D., Mathias, C.J., Dolan, R.J., 2001. Neuroanatomical basis for first- and second-order representations of bodily states. Nat. Neurosci. 4 , 207-212.

Dalgleish, T., 2004. The emotional brain. Nat. Rev., Neurosci. 5, 583-589.

Damasio, A.R., 1996. The somatic marker hypothesis and the possible functions of the prefrontal cortex. Philos. Trans. R. Soc. London, Ser. B Biol. Sci. 351, 1413-1420.

Damasio, A.R., Grabowski, T.J., Bechara, A., Damasio, H., Ponto, L.L., Parvizi, J., Hichwa, R.D., 2000. Subcortical and cortical brain activity during the feeling of self-generated emotions. Nat. Neurosci. 3, $1049-1056$.

Darwin, C., 1872. The Expression of the Emotions in Man and Animals. J. Murray, London.

Davidson, R.J., Irwin, W., Anderle, M.J., Kalin, N.H., 2003. The neural substrates of affective processing in depressed patients treated with venlafaxine. Am. J. Psychiatry 160, 64-75.

Deoni, S.C., Josseau, M.J., Rutt, B.K., Peters, T.M., 2005. Visualization of thalamic nuclei on high resolution, multi-averaged T1 and T2 maps acquired at 1.5 T. Hum. Brain Mapp. 25, 353-359.

Dunckley, P., Wise, R.G., Aziz, Q., Painter, D., Brooks, J., Tracey, I., Chang, L., 2005. Cortical processing of visceral and somatic stimulation: differentiating pain intensity from unpleasantness. Neuroscience 133, $533-542$.

Eysenck, H.J., Eysenck, S.B., 1971. The orthogonality of psychoticism and neuroticism: a factorial study. Percept. Mot. Skills 33, 461-462.

Folkman, S., Lazarus, R.S., Gruen, R.J., DeLongis, A., 1986. Appraisal, coping, health status, and psychological symptoms. J. Pers. Soc. Psychol. 50, 571-579.

Fridlund, A.J., 1991. Evolution and facial action in reflex, social motive, and paralanguage. Biol. Psychol. 32 (1), 3-100.

Gorno-Tempini, M.L., Pradelli, S., Serafini, M., Pagnoni, G., Baraldi, P., Porro, C., Nicoletti, R., Umita, C., Nichelli, P., 2001. Explicit and incidental facial expression processing: an fMRI study. NeuroImage 14, $465-473$.

Gottfried, J.A., O’Doherty, J., Dolan, R.J., 2003. Encoding predictive reward value in human amygdala and orbitofrontal cortex. Science 301, $1104-1107$

Gray, J.E., 1972. Self-rating and Eysenck personality inventory estimates of neuroticism and extraversion. Psychol. Rep. 30, 213-214.

Heimer, L., 2003. A new anatomical framework for neuropsychiatric disorders and drug abuse. Am. J. Psychiatry 160, 1726-1739.

Herwig, U., Abler, B., Walter, H., Erk, S. Expectation of affective stimuliAn fMRI study. Psychiatry Res NeuroImaging, in press.

Horn, K.M., Pong, M., Batni, S.R., Levy, S.M., Gibson, A.R., 2002. Functional specialization within the cat red nucleus. J. Neurophysiol. 87, $469-477$.

Koyama, T., McHaffie, J.G., Laurienti, P.J., Coghill, R.C., 2005. The subjective experience of pain: where expectations become reality. Proc. Natl. Acad. Sci. U. S. A. 102, 12950-12955.

Lang, P.J., 1995. The emotion probe. Studies of motivation and attention. Am. Psychol. 50, 372-385.

Mayberg, H.S., 2003. Modulating dysfunctional limbic-cortical circuits in depression: towards development of brain-based algorithms for diagnosis and optimised treatment. Br. Med. Bull. 65, 193-207.

McClure, S.M., York, M.K., Montague, P.R., 2004. The neural substrates of reward processing in humans: the modern role of FMRI. Neuroscientist $10,260-268$.

Meites, K., Lovallo, W., Pishkin, V., 1980. A comparison of four scales for anxiety, depression, and neuroticism. J. Clin. Psychol. 36, 427-432.

Minamimoto, T., Hori, Y., Kimura, M., 2005. Complementary process to response bias in the centromedian nucleus of the thalamus. Science 308, $1798-1801$

Morel, A., Magnin, M., Jeanmonod, D., 1997. Multiarchitectonic and stereotactic atlas of the human thalamus. J. Comp. Neurol. 387, $588-630$.

Morris, J.S., Friston, K.J., Buchel, C., Frith, C.D., Young, A.W., Calder, A.J., Dolan, R.J., 1998. A neuromodulatory role for the human amygdala in processing emotional facial expressions. Brain 121 (Pt. 1), 47-57.

Nichols, T., Brett, M., Andersson, J., Wager, T., Poline, J.B., 2005. Valid conjunction inference with the minimum statistic. NeuroImage 25, 653-660.

Nimchinsky, E.A., Gilissen, E., Allman, J.M., Perl, D.P., Erwin, J.M., Hof, P.R., 1999. A neuronal morphologic type unique to humans and great apes. Proc. Natl. Acad. Sci. U. S. A. 96, 5268-5273.

Nitschke, J.B., Sarinopoulos, I., Mackiewicz, K.L., Schaefer, H.S., Davidson, R.J., 2006. Functional neuroanatomy of aversion and its anticipation. NeuroImage 29, 106-116.

O’Doherty, J.P., Deichmann, R., Critchley, H.D., Dolan, R.J., 2002. Neural responses during anticipation of a primary taste reward. Neuron 33, $815-826$.

Paulus, M.P., Feinstein, J.S., Castillo, G., Simmons, A.N., Stein, M.B., 2005. Dose-dependent decrease of activation in bilateral amygdala and insula by lorazepam during emotion processing. Arch. Gen. Psychiatry $62,282-288$

Pessoa, L., 2005. To what extent are emotional visual stimuli processed without attention and awareness? Curr. Opin. Neurobiol. 15, 188-196.

Pessoa, L., Kastner, S., Ungerleider, L.G., 2003. NeuroImaging studies of attention: from modulation of sensory processing to top-down control. J. Neurosci. 23, 3990-3998.

Peyron, R., Laurent, B., Garcia-Larrea, L., 2000. Functional imaging of brain responses to pain. A review and meta-analysis. Neurophysiol. Clin. $30,263-288$

Phan, K.L., Wager, T.D., Taylor, S.F., Liberzon, I., 2004. Functional neuroimaging studies of human emotions. CNS Spectr. 9, 258-266.

Phelps, E.A., O'Connor, K.J., Gatenby, J.C., Gore, J.C., Grillon, C., Davis, M., 2001. Activation of the left amygdala to a cognitive representation of fear. Nat. Neurosci. 4, 437-441.

Phillips, M.L., Young, A.W., Senior, C., Brammer, M., Andrew, C., Calder, A.J., Bullmore, E.T., Perrett, D.I., Rowland, D., Williams, S.C., Gray, J.A., David, A.S., 1997. A specific neural substrate for perceiving facial expressions of disgust. Nature 389, 495-498.

Phillips, M.L., Drevets, W.C., Rauch, S.L., Lane, R., 2003. Neurobiology of emotion perception. I: The neural basis of normal emotion perception. Biol. Psychiatry 54, 504-514.

Picardi, A., Caroppo, E., Toni, A., Bitetti, D., Di Maria, G., 2005a. Stability of attachment-related anxiety and avoidance and their relationships with the five-factor model and the psychobiological model of personality. Psychol. Psychother. 78 (Pt. 3), 327-345.

Picardi, A., Toni, A., Caroppo, E., 2005b. Stability of alexithymia and its relationships with the 'big five' factors, temperament, character, and attachment style. Psychother. Psychosom. 74 (6), 371-378.

Ploghaus, A., Tracey, I., Gati, J.S., Clare, S., Menon, R.S., Matthews, P.M., Rawlins, J.N., 1999. Dissociating pain from its anticipation in the human brain. Science 284, 1979-1981.

Sawamoto, N., Honda, M., Okada, T., Hanakawa, T., Kanda, M., Fukuyama, H., Konishi, J., Shibasaki, H., 2000. Expectation of pain enhances responses to nonpainful somatosensory stimulation in the anterior cingulate cortex and parietal operculum/posterior insula: an eventrelated functional magnetic resonance imaging study. J. Neurosci. 20, $7438-7445$

Simmons, A., Matthews, S.C., Stein, M.B., Paulus, M.P., 2004. Anticipation of emotionally aversive visual stimuli activates right insula. NeuroReport $15,2261-2265$.

Smith, C.A., Ellsworth, P.C., 1985. Patterns in cognitive appraisal in emotion. J. Pers. Soc. Psychol. 48 (4), 813-818.

Stoleru, S., Gregoire, M.C., Gerard, D., Decety, J., Lafarge, E., Cinotti, L., Lavenne, F., Le Bars, D., Vernet-Maury, E., Rada, H., Collet, C., Mazoyer, B., Forest, M.G., Magini, F., Spira, A., Comar, D., 1999. Neuroanatomical correlates of visually evoked sexual arousal in human males. Arch. Sex Behav. 28, 1-21. 
Talairach, J., Tournoux, P., 1988. Co-Planar Stereotaxic Atlas of the Human Brain. Thieme, Stuttgart.

Ueda, K., Okamoto, Y., Okada, G., Yamashita, H., Hori, T., Yamawaki, S., 2003. Brain activity during expectancy of emotional stimuli: an fMRI study. NeuroReport 14, 51-55.

Vogt, B.A., 2005. Pain and emotion interactions in subregions of the cingulate gyrus. Nat. Rev., Neurosci. 6, 533-544.

Wager, T.D., Rilling, J.K., Smith, E.E., Sokolik, A., Casey, K.L., Davidson, R.J., Kosslyn, S.M., Rose, R.M., Cohen, J.D., 2004. Placebo-induced changes in FMRI in the anticipation and experience of pain. Science 303, 1162-1167.

Winston, J.S., Strange, B.A., O’Doherty, J., Dolan, R.J., 2002. Automatic and intentional brain responses during evaluation of trustworthiness of faces. Nat. Neurosci. 5, 277-283.
Wood, J.N., Romero, S.G., Knutson, K.M., Grafman, J., 2005. Representation of attitudinal knowledge: role of prefrontal cortex, amygdala and parahippocampal gyrus. Neuropsychologia 43, 249-259.

Yamasaki, H., LaBar, K.S., McCarthy, G., 2002. Dissociable prefrontal brain systems for attention and emotion. Proc. Natl. Acad. Sci. U. S. A. 99 11447-11451.

Yasui, Y., Breder, C.D., Saper, C.B., Cechetto, D.F., 1991. Autonomic responses and efferent pathways from the insular cortex in the rat. $\mathrm{J}$. Comp. Neurol. 303, 355-374.

Zurowski, B., Gostomzyk, J., Gron, G., Weller, R., Schirrmeister, H., Neumeier, B., Spitzer, M., Reske, S.N., Walter, H., 2002. Dissociating a common working memory network from different neural substrates of phonological and spatial stimulus processing. NeuroImage 15, 45-57. 